\section{Insegurança alimentar em comunidades rurais no Nordeste brasileiro: faz diferença ser quilombola?}

\section{Food insecurity in rural communities in Northeast Brazil: does belonging to a slave-descendent community make a difference?}

\section{Inseguridad alimentaria en comunidades rurales en el nordeste brasileño: ¿marca la diferencia ser quilombola?}

\section{Resumo}

Este artigo objetivou identificar a prevalência de insegurança alimentar em uma área rural do Nordeste do Brasil e investigar este desfecho de acordo com a residência em comunidades quilombolas e não quilombolas. Foi um estudo transversal com 21 comunidades rurais, sendo nove quilombolas, em 2014, utilizando a Escala Brasileira de Insegurança Alimentar (EBIA). Foram estimadas prevalências e razões de prevalência para insegurança alimentar, $e$ a análise múltipla foi conduzida por regressão de Poisson com variância robusta. A situação de insegurança alimentar foi encontrada em 52,1\% das famílias estudadas, sendo 64,9\% entre quilombolas e 42\% entre as demais. Insegurança alimentar foi associada a ser quilombola $(R P=1,25)$; ter nível econômico mais baixo $(R P=1,89$; 2,98 e 3,22 para os níveis $C 2, D$ e E, respectivamente); ser beneficiário do programa Bolsa Família $(R P=1,52)$; e ter quatro residentes ou mais no domicílio $(R P=1,20)$. A prevalência de insegurança alimentar foi elevada em toda a população, no entanto as comunidades quilombolas, apesar de pertencerem à mesma área de abrangência das outras comunidades, apresentaram uma prevalência ainda maior de insegurança alimentar, reforçando a vulnerabilidade dessa população.

Segurança Alimentar e Nutricional; Grupo com Ancestrais do

Continente Africano; Origem Étnica e Saúde; População Rural
Etna Kaliane Pereira da Silva 1

Danielle Souto de Medeiros 1

Poliana Cardoso Martins 1

Lillian de Almeida Sousa 1

Gislane Pereira Lima 1

Maria Amanda Sousa Rêgo 1

Tainan Oliveira da Silva 1

Alessandra Silva Freire 1

Fernanda Moitinho Silva 1

doi: 10.1590/0102-311X00005716

\section{Correspondência}

D. S. Medeiros

Rua Rio de Contas, Quadra 17, Lote 58, Vitória da Conquista, BA 45029-094, Brasil.

daniellesoutomedeiros@gmail.com

1 Universidade Federal da Bahia, Vitória da Conquista, Brasil. 


\section{Introdução}

As comunidades quilombolas, reconhecidas como comunidades tradicionais com presunção de ancestralidade negra, estão distribuídas em todo o território nacional, em maior concentração no Nordeste rural do Brasil. Apresentam características heterogêneas com trajetórias históricas próprias. Destacase que tais comunidades são redutos de sobrevivência e resistência física, social e cultural de grupos étnicos marginalizados, em sua maioria negros, desde a época da escravidão até os dias atuais 1,2,3.

Historicamente, sofrem com a invisibilidade e a afronta aos direitos mais básicos de seus moradores. Somente nas últimas décadas, após lutas de vários atores sociais, a população quilombola passa a ser vista, de fato, como sujeitos de direitos, reconhecida na Constituição de 1988, regulamentada no Decreto no 4.8871 de 2003 e em outras políticas públicas voltadas para a essa população 4,5 .

Grupos étnicos de origem quilombola mostram padrões diferenciados de vulnerabilidade quando comparados com a população urbana e rural do país 6 . Apesar de alguns avanços, a melhoria das condições de vida nas comunidades quilombolas caminha a passos lentos. A configuração de vulnerabilidade social nesses territórios ainda vem sendo encontrada com bastante frequência em pesquisas recentes, mostrando a continuidade de um quadro propício para a ocorrência da insegurança alimentar 7 .

A insegurança alimentar é um indicador de iniquidade social e se relaciona à falta de acesso regular e permanente de alimentos em quantidade e qualidade suficientes, ou até mesmo à preocupação com a ausência desse acesso em um futuro próximo, comprometendo a aquisição de outros bens e serviços essenciais 8,9 . Estudos evidenciam que a população rural brasileira possui uma maior prevalência de insegurança alimentar quando comparada à população urbana 10,11.

Em 2006, a Chamada Nutricional Quilombola, realizada com famílias com membros menores de cinco anos, foi a primeira pesquisa que evidenciou a insegurança alimentar vivenciada por essa população em nível nacional 12. Essa realidade se perpetuou e foi reafirmada pela Avaliação da Situação de Segurança Alimentar e Nutricional em Comunidades Quilombolas Tituladas, realizada em 2011, abrangendo 169 comunidades. Foi encontrada uma elevada prevalência de insegurança alimentar (55,6\%), além de precárias condições de vida nos domicílios pesquisados 13 .

Apesar dos estudos sobre insegurança alimentar com a população quilombola, ainda há lacunas do conhecimento a serem preenchidas. Assim, a realização desta pesquisa se orientou por duas questões: 1. Numa mesma região rural, há diferenças na insegurança alimentar entre comunidades quilombolas e não quilombolas? 2. Que fatores poderiam influenciar a ausência de segurança alimentar nesses povos?

Diante do exposto, este trabalho teve como objetivo identificar a prevalência de insegurança alimentar em uma zona rural do Nordeste do Brasil e investigar os fatores associados a esse desfecho, de acordo com a residência em comunidades quilombolas e não quilombolas de uma mesma área de abrangência.

\section{Métodos}

Este foi um estudo transversal, de abordagem domiciliar, que teve como população alvo famílias residentes em uma área rural do Município de Vitória da Conquista, no interior da Bahia. Localizado a $509 \mathrm{~km}$ da capital, na macrorregião econômica do sudoeste. O município está situado numa região de transição entre a caatinga e a mata atlântica, com condições climáticas atípicas. É a terceira maior cidade do estado, com uma população de mais de trezentos mil habitantes, dos quais cerca de $10 \%$ residem na zona rural e, desses, 24,8\% vivem em situação de extrema pobreza (Instituto Brasileiro de Geografia e Estatística. Cidades@. Bahia: Vitória da Conquista.http://cidades.ibge.gov.br/xtras/perfil. php?codmun=293330, acessado em 30/Abr/2016) (Ministério do Desenvolvimento Social e Combate à Fome. Relatório institucional segurança alimentar e nutricional. http://aplicacoes.mds.gov.br/sagi/ portal, acessado em 30/Abr/2015).

A população total da área estudada foi estimada em 1.316 famílias, oriundas de 21 comunidades (sendo nove comunidades remanescentes de quilombos reconhecidas pela Fundação Cultural Palmares - FCP) 2. Essa estimativa foi retirada das fichas A (ficha de cadastramento das famílias) da unidade de saúde da família e confirmada em posterior mapeamento das comunidades. 
No cálculo do tamanho amostral, foram considerados dois estratos populacionais: famílias quilombolas (residentes em comunidades certificadas pela FCP) e não quilombolas. Considerou-se uma prevalência esperada de 35,1\% de insegurança alimentar, conforme resultados da Pesquisa Nacional por Amostra de Domicílios (PNAD 2009) para a zona rural brasileira 14; precisão de 5\%; intervalo de 95\% de confiança (IC95\%) e efeito de desenho igual a 1; ao número resultante foram acrescidos $20 \%$ para possíveis perdas, totalizando uma amostra de 248 e 294 famílias quilombolas e não quilombolas, respectivamente. As famílias foram selecionadas aleatoriamente, de acordo com a distribuição proporcional por comunidade.

Estudo piloto foi realizado em junho de $2014 \mathrm{em}$ uma comunidade rural não participante do estudo principal.

Anteriormente à coleta de dados, foram realizados mapeamento e identificação das famílias, com auxílio dos agentes comunitários de saúde (ACS). Nessa etapa, identificaram-se as coordenadas relativas aos domicílios e equipamentos da comunidade, obtidas em frente à porta principal dos locais. Para o mapeamento, foram utilizados aparelho GPS (Garmin eTrex-30), com precisão de 10m, máquina fotográfica digital, os dados do GPS foram extraídos com o programa EasyGPS (TopoGrafix, Stow, Estados Unidos), versão 5.21, e transferidos para o programa Google Earth (https://www.google. com/earth/), versão 7.1.2.2041.

Simultaneamente ao mapeamento, foi realizada sensibilização com as famílias com a distribuição de material impresso com informações sobre a pesquisa. A pesquisa também foi apresentada na reunião do Conselho Local de Saúde e do Conselho Territorial Quilombola, a fim de obter apoio para realização do estudo.

A coleta de dados ocorreu entre setembro de 2014 e janeiro de 2015. A equipe de entrevistadores foi composta por alunos de graduação, previamente treinados, utilizando computador portátil (HP Pocket Rx5710). Foi construído um manual para o entrevistador, visando padronizar a abordagem e procedimentos, bem como servir de suporte quando do aparecimento de quaisquer dúvidas durante as entrevistas. Para a programação e armazenamento dos dados, foi utilizado o software Questionnaire Development System (QDS; NOVA Research Company, Silver Spring, Estados Unidos), versão 2.6.1.

O indivíduo entrevistado foi o responsável pelo seu grupo familiar (chefe da família), desde que apresentasse idade maior que 18 anos e estivesse apto a responder ao questionário. Na impossibilidade do chefe da família, entrevistou-se outro morador maior de 18 anos. Foram realizadas reentrevistas em aproximadamente $5 \%$ da população amostral, selecionada por sorteio aleatório, para controle de qualidade dos dados.

Utilizou-se como instrumento de coleta um questionário sobre característica sociodemográfica do domicílio e do chefe da família baseado na Pesquisa Nacional de Saúde (http://www.pns.icict.fiocruz. br/arquivos/Domiciliar/Modulo\%20A-PNS.pdf, acessado em 16/Jun/2014), e a Escala Brasileira de Insegurança Alimentar (EBIA), adaptada de Segall-Corrêa \& Marin-León 15.

A insegurança alimentar, variável dependente deste estudo, foi obtida pela EBIA, que é uma escala psicométrica, composta por 15 perguntas fechadas, que une agrupamentos de conceitos, possibilitando estimar as prevalências de insegurança alimentar, e classificá-la de acordo com os níveis de gravidade (leve, moderada e grave). A EBIA avalia a percepção dos entrevistados sobre a alimentação em seu domicilio nos últimos três meses. Ela também possui diferenciação com a presença ou não de moradores menores de 18 anos de idade no domicílio 15.

O estado de segurança alimentar foi definido pelas respostas "não", "não sabe" ou "não se aplica" às primeiras oito perguntas. A presença de uma resposta do tipo "sim" definiu o estado de insegurança alimentar. Tal variável foi também calculada em seus diferentes níveis, segundo o escore obtido, conforme descrito na Tabela 1. Em relação aos pontos foi atribuído 1 ponto para respostas sim, e 0 para respostas não e não sabe 15 .

O estado de segurança alimentar também foi calculado conforme orientado por Hoffmann 16, para fins de comparabilidade com os dados da PNAD 2013 10, utilizando a pontuação descrita na Tabela 1. Para tanto, a resposta à questão 15 , "Nos últimos 3 meses, o(a) senhor(a) perdeu peso porque não tinha dinheiro suficiente para comprar comida?”, foi desprezada, considerando-se a EBIA com apenas 14 questões (Tabela 1). 
Tabela 1

Pontuação para classificação dos domicílios, com e sem menores de 18 anos, nas categorias de segurança alimentar.

\begin{tabular}{|c|c|c|c|c|}
\hline \multirow[t]{2}{*}{ Classificação } & \multicolumn{2}{|c|}{ EBIA com 15 questões } & \multicolumn{2}{|c|}{ EBIA com 14 questões } \\
\hline & $\begin{array}{c}\text { Com menores de } 18 \\
\text { anos }\end{array}$ & $\begin{array}{l}\text { Sem menores de } 18 \\
\text { anos }\end{array}$ & $\begin{array}{c}\text { Com menores de } 18 \\
\text { anos }\end{array}$ & $\begin{array}{c}\text { Sem menores de } \\
\text { 18anos }\end{array}$ \\
\hline Segurança alimentar & 0 & 0 & 0 & 0 \\
\hline Insegurança alimentar leve & 1 a 5 & 1 a 3 & 1 a 5 & 1 a 3 \\
\hline Insegurança alimentar moderada & 6 a 10 & 4 a 6 & 6 a 9 & 4 a 5 \\
\hline Insegurança alimentar grave & 11 a 15 & 7 a 8 & 10 a 14 & 6 a 8 \\
\hline
\end{tabular}

EBIA: Escala Brasileira de Insegurança Alimentar.

Fonte: adaptado de Segall-Corrêa \& Marín-León 15 e Instituto Brasileiro de Geografia e Estatística 10.

As variáveis independentes para a avaliação da insegurança alimentar foram: residência em comunidade quilombola (sim ou não), nível econômico (classificação econômica definida pela Associação Brasileira de Empresas de Pesquisa, níveis B1, B2 e C1, C2, D ou E) 17, participação no programa Bolsa Família (sim ou não), recebimento de benefício de prestação continuada e/ou de outros programa sociais do governo (sim ou não), trabalho mensal fixo de pelo menos um dos moradores do domicílio (sim ou não), escolaridade do chefe da família (não alfabetizado/Fundamental 1 incompleto, Fundamental 1 completo/Fundamental 2 incompleto, Fundamental 2 completo/Médio incompleto ou Médio completo/Ensino Superior), presença de menores de 18 anos no domicílio (sim ou não), número de residentes no domicílio (1 a 3 residentes ou 4 residentes e mais), revestimento das paredes externas do domicílio (alvenaria com revestimento ou outros), tipo de piso (cerâmica/lajota/pedra ou cimento/terra/outros), abastecimento de água (rede geral de distribuição ou outras formas), água canalizada (sim ou não), tratamento da água para beber (filtrada e outras formas ou sem tratamento), número de cômodos no domicílio (1 a 6 cômodos ou 7 cômodos e mais), tipo de fogão utilizado predominantemente (gás de botijão ou lenha e outras formas), existência de geladeira no domicílio (sim ou não), quantidade de banheiro (nenhum, 1 ou 2 e mais), destino do lixo (coletado por serviço de limpeza ou queimado/enterrado/jogado em terreno baldio ou logradouro).

As diferenças entre as proporções foram testadas com a distribuição qui-quadrado de Pearson, qui-quadrado de tendência linear ou teste exato de Fisher para a amostra total e cada estrato (quilombola e não quilombola), como também para comparar os resultados dos níveis de insegurança alimentar obtidos pelas duas escalas (EBIA com 14 questões e EBIA com 15 questões). A prevalência da insegurança alimentar foi estimada em seus diferentes níveis (leve, moderada e grave), no entanto, para as análises bivariadas e multivariadas, a variável 'insegurança alimentar' foi dicotomizada em presença de insegurança alimentar (em qualquer grau: leve, moderada ou grave) e ausência de insegurança alimentar. A razão de prevalência (RP) foi utilizada para estimar a associação da insegurança alimentar e as variáveis explicativas de interesse. Utilizou-se regressão de Poisson com variância robusta, para estimar as RP para a insegurança alimentar, ajustadas por potenciais fatores de confusão para a amostra total e para os estratos quilombola e não quilombola. Foram incluídas no modelo inicial todas as variáveis que, na análise bivariada, apresentaram associação com a insegurança alimentar em nível de significância inferior a 20\%. Para todos os testes e para permanência das variáveis no modelo final, foi utilizado o nível de 5\% de significância. Os modelos foram comparados pelo critério de Akaike. A adequação do modelo foi avaliada pelo qui-quadrado. Utilizou- se o programa Stata (StataCorp LP, College Station, Estados Unidos), versão 12.0 para análise dos dados.

A pesquisa foi aprovada pelo Comitê de Ética em Pesquisa com seres humanos do Instituto Multidisciplinar em Saúde da Universidade Federal da Bahia (CAAE 27003614000005556, em 20 de junho de 2014). Para a coleta dos dados, os participantes foram previamente informados sobre os objetivos da pesquisa, procedimentos e sigilo dos dados com leitura do Termo de Consentimento Livre Esclarecido, expressando sua concordância em participar do estudo pela assinatura ou impressão dactiloscópica. 


\section{Resultados}

Participaram do estudo 459 famílias, sendo 202 quilombolas (44\%) e 257 não quilombolas (56\%). A proporção de perdas (18,5\% para o estrato quilombola e $12,6 \%$ para o não quilombola) foi inferior à prevista inicialmente; o principal motivo foi a ausência de moradores no domicílio após três visitas $(62,2 \%)$. Não houve perdas diferenciais em relação ao número de residentes no domicílio (valor de $\mathrm{p}$ de 0,950 e de 0,952 para os estratos quilombola e não quilombola, respectivamente).

A situação de insegurança alimentar foi encontrada em $52,1 \%$ da população estudada, sendo 30,9\% leve, $15,7 \%$ moderada e 5,4\% grave. Considerando a EBIA com 14 questões, a prevalência de insegurança alimentar foi a mesma, no entanto com $31,4 \%$ leve, $13,1 \%$ moderada e $7,6 \%$ grave. O estrato quilombola exibiu prevalências superiores em todos os níveis de insegurança alimentar quando comparado ao não quilombola. Não houve diferença estatisticamente significativa entre as prevalências de insegurança alimentar de acordo com as escalas (Figura 1).

A maioria das famílias encontrava-se no nível econômico D (48,6\%), 48\% eram beneficiárias do programa Bolsa Família e 6,3\% recebiam Benefício de Prestação Continuada (BPC) ou de outros programas sociais do governo. Cerca de 54,3\% dos domicílios tinham pelo menos um morador com renda proveniente de trabalho mensal fixo e $57,8 \%$ dos chefes das famílias eram não alfabetizados ou tinham nível Fundamental 1 incompleto. Foram identificados 60,4\% dos domicílios com menores de 18 anos e $52 \%$ com 1 a 3 residentes (Tabela 2).

A maior parte das famílias visitadas possuía domicílios com paredes externas de alvenaria com revestimento $(72,1 \%)$, piso de cerâmica, lajota ou pedra $(63,4 \%)$, principal forma de abastecimento de água proveniente da rede geral de distribuição (80,6\%), água canalizada em pelo menos um cômodo (69,7\%), água para beber tratada (83\%), 1 a 6 cômodos $(54,7 \%)$, fogão que utilizava como fonte de energia gás de botijão (64,1\%), geladeira (88,9\%), 1 banheiro $(73,9 \%$ e o lixo era queimado, enterrado ou jogado em terreno baldio (53,6\%) (Tabela 2$)$.

As famílias quilombolas e não quilombolas diferiram significativamente em relação ao: nível econômico, participação no Bolsa Família, ter algum membro com trabalho mensal fixo, escolaridade do chefe da família, tipo de piso no domicílio, existência de água canalizada, tratamento de água para beber, números de cômodos, tipo de fogão utilizado predominantemente, existência de geladeira, quantidade de banheiros e destino do lixo (Tabela 2).

A prevalência de insegurança alimentar em quilombolas foi significativamente superior à observada entre os não quilombolas, sendo de $64,9 \%$ e $42 \%$, respectivamente (Tabela 3). Encontrou-se associação positiva e significativa entre a insegurança alimentar e residir em comunidade quilombola, menor nível econômico (com relação dose-resposta), ser beneficiário do Bolsa Família, presença de menores de 18 anos entre os membros da família, residir em domicílio com quatro residentes e mais, piso de cimento, terra ou outros, ter outras formas de abastecimento de água que não seja a rede geral de distribuição, não ter água canalizada, possuir fogão com fonte de energia de lenha ou outras formas, não ter banheiro, ter lixo queimado, enterrado ou jogado em terreno baldio. Associação negativa e significativa foi constatada com ter trabalho mensal fixo, maior nível de escolaridade do chefe da família (Ensino Fundamental 2 completo e outros), consumir água tratada, domicílio ter 7 cômodos e mais (Tabela 3).

Entre as famílias quilombolas, foi observada associação positiva e significativa com o nível econômico E e ser beneficiário do Bolsa Família, e negativa com residir em domicílio com 7 cômodos ou mais. Entre as famílias não quilombolas, os fatores associados com a insegurança alimentar foram os mesmos que para a amostra total (Tabela 3).

Os seguintes fatores mostraram-se independentemente associados à insegurança alimentar na amostra total: ser quilombola $(\mathrm{RP}=1,25)$; ter nível econômico mais baixo, com gradiente dose-resposta ( $\mathrm{RP}=1,89 ; \mathrm{RP}=2,98$ e $\mathrm{RP}=3,22$ para os níveis $\mathrm{C} 2$, $\mathrm{D}$ e E, respectivamente); ser beneficiário do Bolsa Família $(\mathrm{RP}=1,52)$; e ter 4 residentes e mais no domicílio $(\mathrm{RP}=1,20)$. Para o estrato quilombola, ter nível econômico $\mathrm{D}$ ou $\mathrm{E}(\mathrm{RP}=1,81$ e $\mathrm{RP}=1,95$, respectivamente) e ser beneficiário do Bolsa Família $(\mathrm{RP}=1,28)$ estiveram positivamente associados. Para o não quilombola, verificou-se associação positiva e significativa com nível econômico mais baixo, com gradiente dose-resposta $(\mathrm{RP}=2,25$; $\mathrm{RP}=3,69$ e $\mathrm{RP}=4,09$ para os níveis $\mathrm{C} 2$, $\mathrm{D}$ e E, respectivamente); ser beneficiário do Bolsa Família 


\section{Figura 1}

Prevalência de insegurança alimentar na amostra total e nos estratos quilombola e não quilombola. Bahia, Brasil, 2015

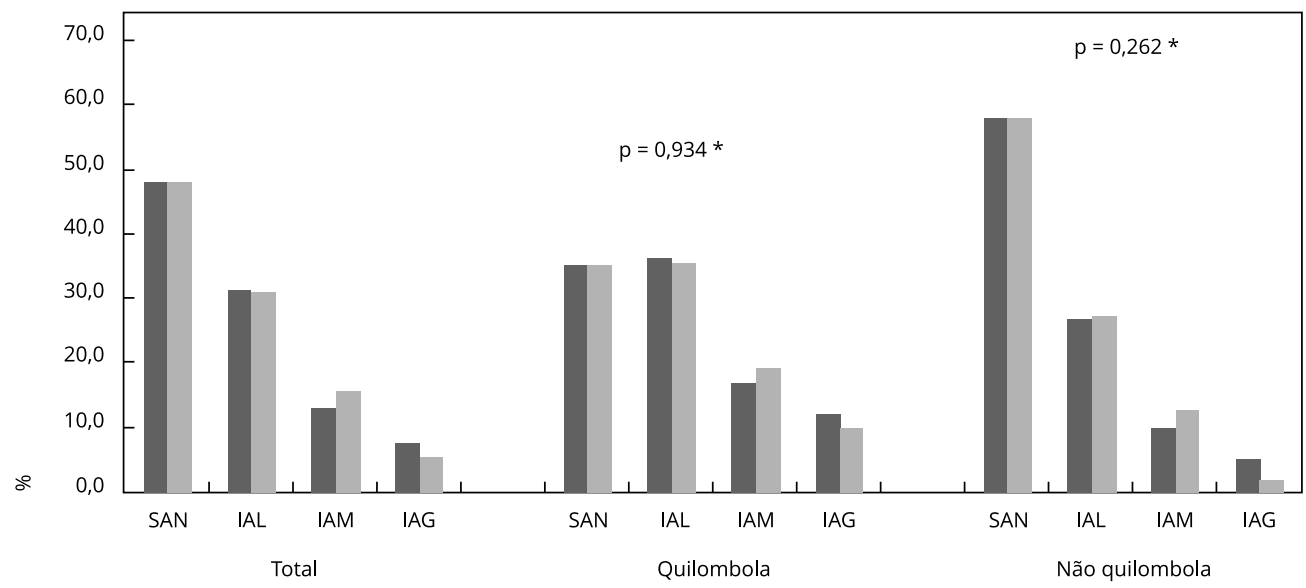

EBIA: Escala Brasileira de Insegurança Alimentar; IAG: insegurança alimentar grave; IAL: insegurança alimentar leve; IAM: insegurança alimentar mediana; SAN: segurança alimentar e nutricional.

* Valor de p calculado pelo teste de qui-quadrado de tendência linear para comparação entre duas estratégias de análise (EBIA 14 questões e EBIA 15 questões).

** Classificação orientada por Hoffmann 16, segundo o Instituto Brasileiro de Geografia e Estatística 10;

*** Classificação segundo Segall-Corrêa \& Marin-León 15.

$(\mathrm{RP}=1,76)$; ter 4 residentes e mais no domicílio $(\mathrm{RP}=1,35)$ e ter fogão que utilizava como fonte de energia lenha e outras formas $(\mathrm{RP}=1,36)$; e associação negativa com consumir água tratada por filtragem ou outra forma $(\mathrm{RP}=0,69)$ (Tabela 4$)$.

\section{Discussão}

A insegurança alimentar esteve presente na maior parte dos domicílios pesquisados, especialmente nas comunidades quilombolas, com prevalências superiores às das populações rurais brasileiras $(35,3 \%)$, da Região Nordeste $(38,1 \%)$ e da Bahia (37,8\%) 10. Tal fato reforça o contexto de vulnerabilidade apresentado por essas famílias, demonstrado pelos baixos níveis econômicos e de escolaridade, grande participação em programas sociais de transferência de renda e precariedade das condições de moradia.

Estudos demostram que as famílias residentes em comunidades rurais têm maior prevalência de insegurança alimentar quando comparadas às famílias residentes na zona urbana 11,18,19. No entanto, Hoffmann 16 discute que apenas o fato de residir na área rural não implica necessariamente maior ocorrência de insegurança alimentar, uma vez que as condições de vida enfrentadas por essa população, que em sua maioria apresenta uma menor renda per capita e escolaridade, podem ser consideradas os principais determinantes desse estado de insegurança.

Um ponto importante a se destacar é que a área rural estudada apresenta condições climáticas desfavoráveis à produção de alimentos, tanto para autoconsumo quanto como fonte de renda. Há chuvas concentradas em curtos períodos do ano, que, aliadas à falta de proteção dos solos, favorece a erosão, e solos bastante degradados, com áreas pedregosas e outras argilosas 20 . A produção também é prejudicada pelo uso de técnicas rudimentares e a quase inexistência de assistência técnica, dificultando o enfrentamento das condições climáticas desfavoráveis. Outro fator relevante é o fato de a 
Tabela 2

Características do domicílio e sociodemográficas da amostra total e dos estratos quilombolas e não quilombolas. Bahia, Brasil, 2015.

\begin{tabular}{|c|c|c|c|c|c|c|c|}
\hline \multirow[t]{2}{*}{ Variáveis } & \multicolumn{2}{|c|}{ Total } & \multicolumn{2}{|c|}{ Quilombola } & \multicolumn{2}{|c|}{ Não quilombola } & \multirow[t]{2}{*}{ Valor de $p$ * } \\
\hline & $\mathbf{n}$ & $\%$ ** & $\mathbf{n}$ & $\%$ ** & $\mathbf{n}$ & $\%$ ** & \\
\hline Nível econômico & & & & & & & 0,000 \\
\hline B1, B2 e C1 & 70 & 15,3 & 18 & 9,0 & 52 & 20,2 & \\
\hline $\mathrm{C} 2$ & 90 & 19,7 & 23 & 11,5 & 67 & 26,1 & \\
\hline D & 222 & 48,6 & 113 & 56,5 & 109 & 42,4 & \\
\hline $\mathrm{E}$ & 75 & 16,4 & 46 & 23,0 & 29 & 11,3 & \\
\hline Participação no programa Bolsa Família & & & & & & & 0,000 \\
\hline Sim & 220 & 48,0 & 121 & 59,9 & 99 & 38,5 & \\
\hline Não & 239 & 52,0 & 81 & 40,1 & 158 & 61,5 & \\
\hline Beneficiárias do BPC ou de outros programas sociais do governo & & & & & & & 0,211 \\
\hline Sim & 29 & 6,3 & 16 & 7,9 & 13 & 5,1 & \\
\hline Não & 430 & 93,7 & 186 & 92,1 & 244 & 94,9 & \\
\hline Trabalho mensal fixo & & & & & & & 0,003 \\
\hline Sim & 249 & 54,3 & 94 & 46,5 & 155 & 60,3 & \\
\hline Não & 210 & 45,8 & 108 & 53,5 & 102 & 39,7 & \\
\hline Escolaridade do chefe da família & & & & & & & 0,000 \\
\hline Não alfabetizado/Fundamental 1 incompleto & 264 & 57,8 & 134 & 67,0 & 130 & 50,6 & \\
\hline Fundamental 1 completo/Fundamental 2 incompleto & 124 & 27,1 & 52 & 26,0 & 72 & 28,0 & \\
\hline Fundamental 2 completo e outros & 69 & 15,1 & 14 & 7,0 & 55 & 21,4 & \\
\hline Presença de moradores menores de 18 anos & & & & & & & 0,120 \\
\hline $\operatorname{Sim}$ & 277 & 60,3 & 130 & 64,4 & 147 & 57,2 & \\
\hline Não & 182 & 39,7 & 72 & 35,7 & 110 & 42,8 & \\
\hline Número de residentes no domicílio & & & & & & & 0,199 \\
\hline 1 a 3 residentes & 239 & 52,1 & 112 & 55,5 & 127 & 49,4 & \\
\hline 4 residentes e mais & 220 & 47,9 & 90 & 44,5 & 130 & 50,6 & \\
\hline Revestimento das paredes externas do domicílio & & & & & & & 0,888 \\
\hline Alvenaria com revestimento & 331 & 72,1 & 145 & 71,8 & 186 & 72,4 & \\
\hline Outros & 128 & 27,9 & 57 & 28,2 & 71 & 27,6 & \\
\hline Tipo de piso & & & & & & & 0,000 \\
\hline Cerâmica, lajota ou pedra & 291 & 63,4 & 104 & 51,5 & 187 & 72,8 & \\
\hline Cimento/Terra/Outros & 168 & 36,6 & 98 & 48,5 & 70 & 27,3 & \\
\hline Abastecimento de água & & & & & & & 0,019 \\
\hline Rede geral de distribuição & 370 & 80,6 & 153 & 75,7 & 217 & 84,4 & \\
\hline Outras formas & 89 & 19,4 & 49 & 24,3 & 40 & 15,6 & \\
\hline Água canalizada & & & & & & & 0,000 \\
\hline Sim & 320 & 69,7 & 112 & 55,5 & 208 & 80,9 & \\
\hline Não & 139 & 30,3 & 90 & 44,5 & 49 & 19,1 & \\
\hline Tratamento da água para beber & & & & & & & 0,000 \\
\hline Filtrada e outras formas & 381 & 83,0 & 150 & 74,3 & 231 & 89,9 & \\
\hline Sem tratamento & 78 & 17,0 & 52 & 25,7 & 26 & 10,1 & \\
\hline Números de cômodos no domicilio & & & & & & & 0,001 \\
\hline 1 a 6 & 251 & 54,7 & 128 & 63,4 & 123 & 47,9 & \\
\hline 7 e mais & 208 & 45,3 & 74 & 36,6 & 134 & 52,1 & \\
\hline Tipo de fogão utilizado predominantemente & & & & & & & 0,000 \\
\hline Gás de botijão & 294 & 64,1 & 89 & 44,1 & 205 & 79,7 & \\
\hline Lenha e outras formas & 165 & 36,0 & 113 & 55,9 & 52 & 20,2 & \\
\hline Existência de geladeira no domicílio & & & & & & & 0,004 \\
\hline $\operatorname{Sim}$ & 408 & 88,9 & 170 & 84,2 & 238 & 92,6 & \\
\hline Não & 51 & 11,1 & 32 & 15,8 & 19 & 7,4 & \\
\hline Quantidade de banheiros & & & & & & & 0,000 \\
\hline 0 & 92 & 20,0 & 58 & 28,7 & 34 & 13,2 & \\
\hline 1 & 339 & 73,9 & 136 & 67,3 & 203 & 79,0 & \\
\hline 2 e mais & 28 & 6,1 & 8 & 4,0 & 20 & 7,8 & \\
\hline Destino do lixo & & & & & & & 0,000 \\
\hline Coletado por serviço de limpeza & 213 & 46,4 & 37 & 18,3 & 176 & 68,5 & \\
\hline Queimado/Enterrado/Jogado em terreno baldio ou logradouro & 246 & 53,6 & 165 & 81,7 & 81 & 31,5 & \\
\hline
\end{tabular}

BPC: Benefício de Prestação Continuada.

* Valor de p calculado pelo teste de qui-quadrado para comparação entre os estratos quilombola e não quilombola;

** Frequência relativa. 
Tabela 3

Prevalência, razão de prevalência e intervalo de 95\% de confiança (IC95\%) da insegurança alimentar, segundo variáveis avaliadas. Bahia, Brasil, 2015.

\begin{tabular}{|c|c|c|c|c|c|c|}
\hline \multirow[t]{2}{*}{ Variáveis } & \multicolumn{2}{|r|}{ Total } & \multicolumn{2}{|c|}{ Quilombola } & \multicolumn{2}{|c|}{ Não quilombola } \\
\hline & $\mathbf{P}(\%)$ & RP (IC95\%) & $\mathbf{P}(\%)$ & RP (IC95\%) & $P(\%)$ & RP (IC95\%) \\
\hline Quilombola & \multicolumn{2}{|r|}{$p^{*}=0,000$} & \multicolumn{2}{|r|}{ - } & \multicolumn{2}{|r|}{-} \\
\hline Sim & 64,9 & $1,54(1,29 ; 1,84)$ & - & - & - & - \\
\hline Não & 42,0 & 1,00 & - & - & - & - \\
\hline Nível econômico & \multicolumn{2}{|c|}{$p^{*}=0,000$} & \multicolumn{2}{|r|}{$p^{*}=0,027$} & \multicolumn{2}{|r|}{$p^{*}=0,000$} \\
\hline B1, B2 e C1 & 18,6 & 1,00 & 38,8 & 1,00 & 11,5 & 1,00 \\
\hline $\mathrm{C} 2$ & 35,6 & $1,91(1,09 ; 3,37)$ & 52,2 & $1,34(0,66 ; 2,70)$ & 29,8 & $2,58(1,12 ; 5,99)$ \\
\hline D & 63,1 & $3,40(2,06 ; 5,61)$ & 68,1 & $1,75(0,96 ; 3,17)$ & 57,8 & $5,00(2,32 ; 10,83)$ \\
\hline E & 70,7 & $3,80(2,28 ; 6,35)$ & 73,9 & $1,90(1,04 ; 3,49)$ & 65,5 & $5,67(2,55 ; 12,63)$ \\
\hline Participação no programa Bolsa Família & \multicolumn{2}{|c|}{$p *=0,000$} & \multicolumn{2}{|c|}{$p *=0,024$} & \multicolumn{2}{|r|}{$p *=0,000$} \\
\hline Sim & 69,0 & $1,90(1,57 ; 2,30)$ & 71,1 & $1,27(1,02 ; 1,60)$ & 66,7 & $2,50(1,86 ; 3,36)$ \\
\hline Não & 36,4 & 1,00 & 55,6 & 1,00 & 26,6 & 1,00 \\
\hline Beneficiárias do BPC ou de outros programas sociais do governo & \multicolumn{2}{|c|}{$p *=0,265$} & \multicolumn{2}{|c|}{$p *=0,734$} & \multicolumn{2}{|r|}{$p^{*}=0,375$} \\
\hline Sim & 62,1 & $1,20(0,90 ; 1,63)$ & 68,7 & $1,06(0,75 ; 1,50)$ & 53,8 & $1,30(0,77 ; 2,20)$ \\
\hline Não & 51,4 & 1,00 & 64,5 & 1,00 & 41,4 & 1,00 \\
\hline Trabalho mensal fixo & & $p *=0,010$ & & $*=0,777$ & & $p *=0,018$ \\
\hline Sim & 46,6 & $0,79(0,67 ; 0,95)$ & 63,8 & $0,97(0,79 ; 1,19)$ & 36,1 & $0,71(0,53 ; 0,94)$ \\
\hline Não & 58,6 & 1,00 & 65,7 & 1,00 & 51,0 & 1,00 \\
\hline Escolaridade do chefe da família & & $p *=0,000$ & & $*=0,059$ & & $p^{*}=0,000$ \\
\hline Não alfabetizado/Fundamental 1 incompleto & 58,7 & 1,00 & 67,2 & 1,00 & 50,0 & 1,00 \\
\hline Fundamental 1 completo/Fundamental 2 incompleto & 54,8 & $0,93(0,77 ; 1,12)$ & 67,3 & $1,00(0,80 ; 1,25)$ & 45,8 & $0,91(0,67 ; 1,24)$ \\
\hline Fundamental 2 completo e outros & 21,7 & $0,37(0,23 ; 0,58)$ & 35,7 & $0,53(0,26 ; 1,09)$ & 18,2 & $0,36(0,20 ; 0,65)$ \\
\hline Presença de moradores menores de 18 anos & & $p *=0,005$ & & $*=0,149$ & & $p *=0,036$ \\
\hline Sim & 57,4 & $1,30(1,08 ; 1,58)$ & 68,5 & $1,17(0,93 ; 1,47)$ & 47,6 & $1,38(1,01 ; 1,87)$ \\
\hline Não & 44,0 & 1,00 & 58,3 & 1,00 & 34,5 & 1,00 \\
\hline Número de residentes no domicílio & & $p *=0,001$ & & $*=0,095$ & & $p^{*}=0,001$ \\
\hline 1 a 3 & 44,8 & 1,00 & 59,8 & 1,00 & 31,5 & 1,00 \\
\hline 4 e mais & 60,0 & $1,34(1,12 ; 1,60)$ & 71,1 & $1,19(0,97 ; 1,45)$ & 52,3 & $1,66(1,22 ; 2,25)$ \\
\hline Revestimento das paredes externas do domicílio & & $p *=0,365$ & & $*=0,320$ & & $p *=0,742$ \\
\hline Alvenaria com revestimento & 50,8 & $0,91(0,76 ; 1,10)$ & 62,8 & $0,89(0,72 ; 1,10)$ & 41,4 & $0,95(0,69 ; 1,30)$ \\
\hline Outros & 55,5 & 1,00 & 70,2 & 1,00 & 43,6 & 1,00 \\
\hline Tipo de piso & & $p *=0,001$ & & $*=0,471$ & & $p^{*}=0,015$ \\
\hline Cerâmica, lajota ou pedra & 46,4 & 1,00 & 62,5 & 1,00 & 37,4 & 1,00 \\
\hline Cimento/Terra/Outros & 62,0 & $1,33(1,12 ; 1,58)$ & 67,4 & $1,07(0,87 ; 1,32)$ & 54,3 & $1,45(1,09 ; 1,92)$ \\
\hline Abastecimento de água & & $p *=0,022$ & & $*=0,445$ & & $p^{*}=0,070$ \\
\hline Rede geral de distribuição & 49,5 & 1,00 & 63,4 & 1,00 & 36,6 & 1,00 \\
\hline Outras formas & 63,0 & $1,27(1,05 ; 1,54)$ & 69,4 & $1,09(0,88 ; 1,37)$ & 55,0 & $1,38(1,00 ; 1,92)$ \\
\hline Água canalizada & & $p *=0,000$ & & $*=0,095$ & & $p^{*}=0,000$ \\
\hline Sim & 44,4 & 1,00 & 59,8 & 1,00 & 36,1 & 1,00 \\
\hline Não & 69,8 & $1,57(1,33 ; 1,85)$ & 71,1 & $1,18(0,97 ; 1,45)$ & 67,3 & $1,87(1,43 ; 2,44)$ \\
\hline Tratamento da água para beber & & $p *=0,005$ & & $*=0,667$ & & $p *=0,011$ \\
\hline Filtrada e outras formas & 49,0 & $0,73(0,61 ; 0,89)$ & 64,0 & $0,95(0,76 ; 1,19)$ & 39,4 & $0,60(0,43 ; 0,83)$ \\
\hline Sem tratamento & 66,7 & 1,00 & 67,3 & 1,00 & 65,4 & 1,00 \\
\hline Números de cômodos no domicílio & & $p^{*}=0,000$ & & $*=0,015$ & & $p^{*}=0,009$ \\
\hline 1 a 6 & 61,0 & 1,00 & 71,1 & 1,00 & 50,4 & 1,00 \\
\hline 7 e mais & 41,4 & $0,67(0,56 ; 0,82)$ & 54,1 & $0,76(0,59 ; 0,96)$ & 34,3 & $0,68(0,50 ; 0,91)$ \\
\hline Tipo de fogão utilizado predominantemente & & $p *=0,000$ & & $*=0,270$ & & $p^{*}=0,000$ \\
\hline Gás de botijão & 43,5 & 1,00 & 60,7 & 1,00 & 36,1 & 1,00 \\
\hline Lenha e outras formas & 67,3 & $1,55(1,31 ; 1,83)$ & 68,1 & $1,12(0,91 ; 1,38)$ & 65,4 & $1,81(1,38 ; 2,37)$ \\
\hline Existência de geladeira no domicílio & & $p *=0,306$ & & $*=0,479$ & & $p *=0,145$ \\
\hline Sim & 51,2 & $0,87(0,68 ; 1,12)$ & 65,9 & $1,10(0,82 ; 1,50)$ & 40,8 & $0,70(0,46 ; 1,06)$ \\
\hline Não & 58,8 & 1,00 & 59,4 & 1,00 & 57,9 & 1,00 \\
\hline Quantidade de banheiros & & $p *=0,000$ & & $*=0,022$ & & $p^{*}=0,001$ \\
\hline 0 & 74,0 & $2,95(1,54 ; 5,68)$ & 77,6 & $1,63(0,66 ; 4,03)$ & 67,6 & $1,99(0,81 ; 4,87)$ \\
\hline 1 & 48,4 & $1,94(1,00 ; 3,71)$ & 61,0 & $2,07(0,83 ; 5,13)$ & 39,9 & $3,38(1,36 ; 8,39)$ \\
\hline 2 e mais & 25,0 & 1,00 & 37,5 & 1,00 & 20,0 & 1,00 \\
\hline Destino do lixo & & $p *=0,000$ & & $*=0,998$ & & $p^{*}=0,000$ \\
\hline Coletado por serviço de limpeza & 40,0 & 1,00 & 64,9 & 1,00 & 34,6 & 1,00 \\
\hline $\begin{array}{l}\text { Queimado/Enterrado/Jogado em terreno baldio ou } \\
\text { logradouro }\end{array}$ & 62,7 & $1,56(1,30 ; 1,90)$ & 64,8 & $0,99(0,77 ; 1,30)$ & 58,0 & $1,67(1,27 ; 2,20)$ \\
\hline
\end{tabular}

BPC: Benefício de Prestação Continuada; RP: razão de prevalência; P: prevalência de insegurança alimentar.

* Valor de p calculado pelo teste de qui-quadrado. 


\section{Tabela 4}

Razões de prevalências (RP) ajustadas para insegurança alimentar das variáveis incluídas no modelo final de regressão. Bahia, Brasil, 2015.

\begin{tabular}{|c|c|c|c|c|c|c|}
\hline \multirow[t]{2}{*}{ Variáveis } & \multicolumn{2}{|c|}{ Total } & \multicolumn{2}{|c|}{ Quilombola } & \multicolumn{2}{|c|}{ Não quilombola } \\
\hline & $\mathbf{R P}$ & IC95\% & $\mathbf{R P}$ & IC95\% & $\mathbf{R P}$ & IC95\% \\
\hline \multicolumn{7}{|l|}{ Quilombola } \\
\hline Sim & 1,25 & 1,$06 ; 1,47$ & - & - & - & - \\
\hline Não & 1,00 & - & - & - & - & - \\
\hline \multicolumn{7}{|l|}{ Níveis econômicos } \\
\hline B1, B2 e C1 & 1,00 & - & 1,00 & - & 1,00 & - \\
\hline $\mathrm{C} 2$ & 1,89 & 1,$11 ; 3,23$ & 1,39 & 0,$70 ; 2,73$ & 2,25 & 1,$02 ; 4,97$ \\
\hline $\mathrm{D}$ & 2,98 & 1,$84 ; 4,84$ & 1,81 & 1,$02 ; 3,20$ & 3,69 & 1,$74 ; 7,84$ \\
\hline$E$ & 3,22 & 1,$95 ; 5,32$ & 1,95 & 1,$08 ; 3,49$ & 4,09 & 1,$87 ; 8,93$ \\
\hline \multicolumn{7}{|c|}{ Participação no programa Bolsa Família } \\
\hline $\operatorname{Sim}$ & 1,52 & 1,$25 ; 1,84$ & 1,28 & 1,$03 ; 1,59$ & 1,76 & 1,$31 ; 2,37$ \\
\hline Não & 1,00 & - & 1,00 & - & 1,00 & - \\
\hline \multicolumn{7}{|c|}{ Número de residentes no domicílio } \\
\hline 1 a 3 & 1,00 & - & - & - & 1,00 & - \\
\hline 4 e mais & 1,20 & 1,$01 ; 1,43$ & - & - & 1,35 & 1,$02 ; 1,79$ \\
\hline \multicolumn{7}{|c|}{ Tratamento da água para beber } \\
\hline Filtrada e outras formas & - & - & - & - & 0,69 & 0,$49 ; 0,97$ \\
\hline Sem tratamento & - & - & - & - & 1,00 & - \\
\hline \multicolumn{7}{|c|}{ Tipo de fogão utilizado predominantemente } \\
\hline Gás de botijão & - & - & - & - & 1,00 & - \\
\hline Lenha e outras formas & - & - & - & - & 1,36 & 1,$07 ; 1,74$ \\
\hline
\end{tabular}

IC95\%: intervalo de 95\% de confiança.

maioria das propriedades ser adquirida por meio de herança familiar, portanto repartidas ao longo do tempo, resultando em pequenas propriedades com até 3 hectares, nas quais se produz em pequena quantidade feijão, milho e mandioca. Outra dificuldade é com o transporte, tanto de pessoas para o acesso a bens e serviços, como também para escoamento da produção ${ }^{20}$. Como as atividades agrícolas são uma das principais fontes de renda dessas famílias, essas características influenciam no aumento da vulnerabilidade delas.

A insegurança alimentar acontece desde a preocupação com a possível falta de alimentos em um futuro próximo até a fome propriamente dita. Com essa amplitude de possibilidades, faz-se a necessidade da classificação em níveis de insegurança alimentar. A insegurança alimentar leve é caracterizada pela preocupação quanto ao acesso aos alimentos no futuro e a qualidade inadequada da alimentação. Já a moderada, pela restrição quantitativa de alimentos entre os adultos; e a grave, com a redução da quantidade de alimentos entre as crianças e a presença da fome (quando alguém fica o dia inteiro sem comer por falta de dinheiro para comprar alimentos) 10.

Nas famílias estudadas, percebe-se que uma porcentagem considerável da população convive com a fome. Ao comparar com a PNAD 2013 10, as frequências de insegurança alimentar leve, moderada e grave para a zona rural brasileira $(21,4 \%, 8,4 \%$ e 5,5\%, respectivamente), para a população da Região Nordeste $(23,6 \%, 8,9 \%$ e 5,6\%) e para a Bahia $(21,8 \%, 9,4 \%$ e 6,6\%) foram inferiores às encontradas neste estudo, demostrando que a população estudada não alcançou os mesmos avanços na melhoria da segurança alimentar, encontrados em nível nacional. Essa situação é ainda mais grave entre os quilombolas, nos quais a insegurança alimentar grave atinge o valor de $12 \%$.

As famílias quilombolas apresentaram prevalência $25 \%$ maior de insegurança alimentar que as não quilombolas. A estratificação de famílias pertencentes a uma mesma área de abrangência evidencia que as famílias quilombolas, mesmo na atualidade, possuem desigualdades nas condições de vida e acesso aos alimentos em relação a famílias não quilombolas. Esse fato leva a concluir que outros 
fatores que não apenas a localização em zona rural e o difícil acesso a essas comunidades podem influenciar nas condições inadequadas de vida e alimentação. Em 2011, Bezerra et al. 21, em pesquisa com comunidades quilombolas de Vitória da Conquista, já relatavam menor cobertura de abastecimento de água por rede geral de distribuição e de coleta regular de lixo, baixo grau de escolaridade e renda per capita familiar, quando comparada com a população total do município, do estado e do país. Condições de vida desiguais são reafirmadas no presente estudo. $\mathrm{O}$ distinto grau de vulnerabilidade encontrado pela medida da insegurança alimentar, reforça a possibilidade da utilização da insegurança alimentar como um indicador de iniquidade social, conforme ressaltaram Panigassi et al. 9 .

A renda familiar é entendida como um dos determinantes mais importantes da insegurança alimentar 16, tanto que a própria EBIA é um instrumento que, na sua construção, já identifica a insegurança alimentar ocasionada pela falta de dinheiro. Isso se dá porque, no Brasil, o acesso aos alimentos ocorre geralmente por meio da compra 16.

As famílias de zona rural geralmente não possuem renda fixa, assim a classificação em nível econômico expressa uma forma mais apropriada à situação delas, pois estima a condição econômica da família baseada nos bens do domicílio (geladeira, rádio, motocicleta, máquina de lavar roupas, dentre outros), na presença de empregado doméstico mensalista e na escolaridade do chefe da família. Os níveis econômicos apresentaram associação independente com a insegurança alimentar, com efeito de dose-resposta, reforçando a sua relação de causalidade, quanto menor o nível econômico maior a prevalência de insegurança alimentar. No estrato quilombola, esse efeito foi observado apenas para os níveis $\mathrm{D}$ e $\mathrm{E}$ e com magnitude menor que o estrato não quilombola, provavelmente em função da maior homogeneidade dos dados. Além disso, a EBIA, por ser uma escala psicométrica com elementos subjetivos, pode sofrer alterações na medida de insegurança alimentar em famílias extremamente pobres, dentre as quais a ausência de alimentos pode não ser declarada, por ter se tornado "comum" 3 . Outros estudos 22,23,24,25 também encontraram essa relação da insegurança alimentar com os níveis econômicos.

A escolaridade do chefe da família se mostrou significativa na primeira análise, porém essa variável não permaneceu no modelo final. Esse resultado difere do encontrado em estudo com famílias dos municípios do norte de Alagoas 19 e entre trabalhadores de restaurantes populares do Rio de Janeiro 26; contudo se assemelha ao achado entre beneficiários do Bolsa Família em Toledo (Paraná) 27, famílias com adolescentes na Amazônia Legal Brasileira 28, usuários de restaurantes populares no Brasil 29 e famílias residentes em uma área de abrangência do Núcleo de Apoio à Saúde da Família em Itumbiara (Goiás) 23. Como neste trabalho, foi utilizado o nível econômico do domicílio, o qual considera a escolaridade do chefe da família para a sua construção. Isso pode ter contribuído para a não manutenção dessa última na análise ajustada. Outra questão importante é a homogeneidade dessa variável (84,9\% não tinham o Ensino Fundamental completo, proporção mais alta entre as famílias quilombolas - 93\%).

A prevalência de insegurança alimentar foi mais elevada em domicílios com 4 residentes e mais, sendo $20 \%$ mais prevalente que em domicílios com 1 a 3 residentes, resultado que pode ser corroborado por outros estudos 19,23,27. Aschau et al. 27 explicam que famílias numerosas necessitam de mais recursos para a aquisição de alimentos e que o aumento da renda nem sempre acompanha o crescimento familiar.

A participação no Bolsa Família, programa brasileiro de transferência condicionada de renda, mostra-se uma importante forma de redução da desigualdade social 30 e contribui para o aumento do acesso aos alimentos e variedade deles nos lares dos beneficiários 31. Cabral et al. 32, em uma coorte que avaliou a participação no Bolsa Família e a segurança alimentar nos anos de 2005 e 2011, em municípios do interior da Paraíba, apontaram que o programa contribui no incremento da renda e no aumento da segurança alimentar e insegurança leve em detrimento da insegurança moderada ou grave, mas, ainda assim indica que outras variáveis sociais podem estar relacionadas com esse resultado.

Entretanto, estudos ainda encontram altas prevalências de insegurança alimentar entre as famílias beneficiárias 19,23,27,31,33. Da mesma forma, prevalência elevada de insegurança alimentar (69\%) foi encontrada entre os beneficiários do presente estudo, tanto para famílias quilombolas quanto para não quilombolas, e a participação no Bolsa Família mostrou-se independentemente associada à insegurança alimentar nessas famílias, mesmo após ajuste. Como este foi um estudo transversal em que as variáveis explicativas e o desfecho são mensurados num mesmo momento, não se pode descartar a 
possibilidade de causalidade reversa, dado que o estado de insegurança alimentar pode ter antecedido o recebimento do benefício. Assim, as famílias em situação de maior vulnerabilidade, e consequente insegurança alimentar, podem, por esse motivo, ter sido contempladas no programa.

Ainda assim, esses fatos evidenciam que o programa, mesmo melhorando as condições de vida das famílias, por si só, não consegue garantir a segurança alimentar delas. Outra questão é que apesar de o Bolsa Família aumentar o acesso e a variedade quantitativa dos alimentos, não é necessariamente acompanhado de melhoria na qualidade nutricional da alimentação 31,34,35.

Os programas de transferência condicionada de renda são importantes para a garantia da segurança alimentar. Todavia, devem ser associados com outras políticas públicas de educação alimentar e nutricional, programas de infraestrutura e ações que impactem o sistema produtivo (como geração de emprego, formalização do trabalho, estabilidade dos preços dos alimentos e apoio à agricultura familiar), entre outras ações 34 , visando à melhoria no acesso quantitativo aos alimentos, mas também à possibilidade de escolha de uma alimentação saudável e adequada, respeitando o meio ambiente e a cultura alimentar, com valorização dos alimentos regionais.

O presente estudo apresenta algumas limitações. Em razão do baixo grau de escolaridade de uma grande parcela da população estudada, pode ter ocorrido dificuldades na compreensão das questões da EBIA, levando a um viés de informação. A fim de minimizar esse efeito, foram realizados treinamento dos entrevistadores, estudo piloto e a utilização de um manual para o entrevistador, contendo maiores explicações sobre as questões para um melhor entendimento e padronização da coleta de dados.

Como este estudo foi realizado no meio rural, a prevalência de insegurança alimentar também pode sofrer influências da sazonalidade, por conta da maior oferta de trabalho temporário em períodos de colheitas e da maior produção de alimentos pelas famílias. Contudo, é razoável supor que a vulnerabilidade da ocorrência de insegurança alimentar nessa população não seja alterada.

\section{Considerações finais}

As famílias da zona rural estudada vivenciam uma situação de elevada prevalência de insegurança alimentar. Nesse contexto, dada a sua vulnerabilidade, as famílias quilombolas ganham destaque que se reflete inclusive no estado de insegurança. Ser quilombola não apenas está associado a um aumento da prevalência da insegurança alimentar, mas também à maior gravidade de seus níveis, em comparação com famílias não quilombolas que residem numa mesma área geográfica.

Apesar dos avanços nas ações afirmativas e de políticas públicas voltadas para essa população, ainda há muito a ser conquistado. Os resultados deste trabalho demonstram a maior vulnerabilidade econômica, social e de moradia das famílias quilombolas.

Considerando ser uma população residente na área rural, esse pode ser um ambiente favorável para a produção de alimentos de forma social, econômica e ambientalmente sustentável, que favoreceria $o$ acesso regular e permanente aos alimentos em quantidade e qualidade suficientes, baseada em práticas alimentares seguras. No entanto, é necessária a melhoria das condições de vida dessas populações com maior acesso a serviços de educação e saúde. Políticas públicas de incentivo à produção agroecológica (regulamentação fundiária, assistência técnica, distribuição de sementes, acesso à água, entre outros) e de educação alimentar e nutricional que valorizem os alimentos regionais, são também importantes.

A atuação da equipe de saúde da família, no âmbito da atenção primária em saúde, é uma estratégia de grande valia para o fortalecimento e qualificação do cuidado alimentar e nutricional nas comunidades rurais e quilombolas. Todavia, é importante destacar que as ações devem ser planejadas com intuito de atender às especificidades dessas populações. 


\section{Colaboradores}

E. K. P. Silva participou da implementação do estudo, coleta de dados, interpretação dos resultados e redação do artigo. D. S. Medeiros e L. A. Sousa colaboraram na concepção do projeto e implementação do estudo, interpretação dos resultados e redação do artigo. P. C. Martins contribuiu na interpretação dos resultados e análise crítica relevante do conteúdo intelectual. G. P. Lima, M. A. S. Rêgo, T. O. Silva, A. S. Freire e F. M. Silva participaram da implementação do estudo, coleta de dados, interpretação dos resultados e análise crítica relevante do conteúdo intelectual. Os autores são responsáveis por todos os aspectos do trabalho na garantia de exatidão e integridade de qualquer parte da obra.

\section{Agradecimentos}

Aos entrevistadores, agentes comunitários de saúde e demais profissionais das equipes de saúde da família, fundamentais para a execução deste trabalho. Às famílias que participaram da pesquisa pela receptividade e colaboração. Ao Pró-Saúde/PET Saúde - Edital no 24, de 15 de dezembro de 2011, Ministério da Saúde.

\section{Referências}

1. Brasil. Decreto no 4.887 , de 20 de novembro de 2003. Regulamenta o procedimento para identificação, reconhecimento, delimitação, demarcação e titulação das terras ocupadas por remanescentes das comunidades dos quilombos de que trata o art. 68 do Ato das Disposições Constitucionais Transitórias. Diário Oficial da União 2003; 21 nov.

2. Fundação Cultural Palmares. Comunidades quilombolas de Vitória da Conquista, Bahia. http://www.palmares.gov.br/?page_id=88\# (acessado em 16/Jun/2014).

3. Silva VRR. Comunidades quilombolas, racismo ambiental e conflitos territoriais. In: Pinto AR, Borges JC, Novo MP, Pires PS, organizadores. Quilombos do Brasil: segurança alimentar e nutricional em territórios titulados. Brasília: Ministério do Desenvolvimento Social e Combate à Fome; 2014. p. 151-69. (Cadernos de Estudos de Desenvolvimento Social em Debate, 20).
4. Leite IB. O projeto político quilombola: desafios, conquistas e impasses atuais. Revista Estudos Feministas 2008;16:965-77.

5. Secretaria Especial de Políticas de Promoção da Igualdade Racial. Programa Brasil Quilombola. Brasília: Editorial Abaré; 2005.

6. Guerrero AFH, Oliveira e Silva D, Toledo LM, Guerrero JCH, Teixeira P. Mortalidade infantil em remanescentes de quilombos do Munícipio de Santarém - Pará, Brasil. Saúde Soc 2007; 16:103-10.

7. Brandão A, Jorge AL. Comunidades quilombolas, acesso a programas sociais e segurança alimentar e nutricional. In: Rocha C, Burlandy L, Magalhães R, organizadores. Segurança alimentar e nutricional: perspectivas, aprendizados e desafios para as políticas públicas. Rio de Janeiro: Editora Fiocruz; 2013. p. 213-25. 
8. Brasil. Lei no 11.346 , de 15 de setembro de 2006. Cria o Sistema Nacional de Segurança Alimentar e Nutricional - SISAN com vistas em assegurar o direito humano à alimentação adequada e dá outras providências. Diário Oficial da União 2006; 15 set.

9. Panigassi G, Segall-Corrêa AM, Marin-León L, Pérez-Escamilla R, Sampaio MFA, Maranha LK. Insegurança alimentar como indicador de iniquidade: análise de inquérito populacional. Cad Saúde Pública 2008; 24:2376-84

10. Instituto Brasileiro de Geografia e Estatística. Pesquisa Nacional por Amostra de Domicílios: segurança alimentar, 2013. Rio de Janeiro: Instituto Brasileiro de Geografia e Estatística; 2014.

11. Rocha EMB, Lima RT, Almeida PC. Insegurança alimentar relacionada à área de residência em município do Semiárido brasileiro. Cad Saúde Colet (Rio J.) 2014; 22:205-11.

12. Ministério do Desenvolvimento Social e Agrário. Chamada nutricional quilombola 2006. http://www.mds.gov.br/gestaodainformacao/ disseminacao/sumarios-executivos-de-pes quisas $/ 2007 /$ chamada-nutricional-quilombo la-2006/chamada-nutricional-quilombola-2006 (acessado em 20/Jan/2013).

13. Sardinha LMV, Campos R, Pires OS, Jannuzzi P. Análise das condições de vida, segurança alimentar e nutricional e acesso a programas sociais em comunidades quilombolas tituladas. In: Pinto AR, Borges JC, Novo MP, Pires PS, organizadores. Quilombos do Brasil: segurança alimentar e nutricional em territórios titulados. Brasília: Ministério do Desenvolvimento Social e Combate à Fome; 2014. p. 3152. (Cadernos de Estudos de Desenvolvimento Social em Debate, 20).

14. Instituto Brasileiro de Geografia e Estatística. Pesquisa Nacional por Amostra de Domicílios 2004/2009: segurança alimentar. Rio de Janeiro: Instituto Brasileiro de Geografia e Estatística; 2010.

15. Segall-Corrêa AM, Marin-León L. A segurança alimentar no Brasil: proposição e usos da escala brasileira de medida da insegurança alimentar (EBIA) de 2003 a 2009. Segurança Alimentar e Nutricional 2009; 16:1-19.

16. Hoffmann R. Brasil, 2013: mais segurança alimentar. Segurança Alimentar e Nutricional 2014; 21:422-36.

17. Associação Brasileira de Empresas de Pesquisa. Critério de classificação econômica no Brasil. São Paulo: Associação Brasileira de Empresas de Pesquisa; 2014.

18. Vianna RPT, Segall-Corrêa AM. Insegurança alimentar das famílias residentes em municípios do interior do estado da Paraíba, Brasil. Rev Nutr 2008; 21 Suppl:111s-22s.

19. Ferreira HS, Souza MEDCA, Moura FA, Horta BL. Prevalência e fatores associados à insegurança alimentar e nutricional em famílias dos municípios do norte de Alagoas, Brasil, 2010. Ciênc Saúde Coletiva 2014; 19:1533-42.
20. Maia MR. Sustentabilidade e agricultura familiar em Vitória da Conquista - BA [Tese de Doutorado]. Aracaju: Universidade Federal de Sergipe; 2012.

21. Bezerra VM, Medeiros DS, Gomes KO, Souzas R, Giatti L, Steffens AP, et al. Inquérito de saúde em comunidades quilombolas de Vitória da Conquista, Bahia, Brasil (Projeto COMQUISTA): aspectos metodológicos e análise descritiva. Ciênc Saúde Coletiva 2014; 19:1835-47.

22. Santos JV, Gigante DP, Domingues MR. Prevalência de insegurança alimentar em Pelotas, Rio Grande do Sul, Brasil, e estado nutricional de indivíduos que vivem nessa condição. Cad Saúde Pública 2010; 26:41-9.

23. Peixoto MRG, Ramos K, Martins KA, Schincaglia RM, Braudes-Silva LA. Insegurança alimentar na área de abrangência do Núcleo de Apoio à Saúde da Família em Itumbiara, Goiás. Epidemiol Serv Saúde 2014; 23:327-36.

24. Bastos CMM, Pinheiro ARO, Gubert MB. Insegurança alimentar e nutricional e fatores associados em famílias do Núcleo Rural Agrícola Lamarão, no Distrito Federal. Tempus (Brasília) 2014; 8:133-56.

25. Nascimento AL, Gonçalves FCLSP, Maia SR, Schneider S, Lira PIC. A construção de capacidades e meios de vida na garantia da segurança alimentar do rural pernambucano. Segurança Alimentar e Nutricional 2012; 19:50-62.

26. Falcão ACML, Aguiar OB, Fonseca MJM. Association of socioeconomic, labor and health variables related to food insecurity in workers of the Popular Restaurants in the city of Rio de Janeiro. Rev Nutr 2015; 28:77-87.

27. Anschau FR, Matsuo T, Segall-Corrêa AM. Insegurança alimentar entre beneficiários de programas de transferência de renda. Rev Nutr 2012; 25:177-89.

28. Guerra LDS, Espinosa MM, Bezerra ACD, Guimarães LV, Lima-Lopes MA. Insegurança alimentar em domicílios com adolescentes da Amazônia Legal Brasileira: prevalência e fatores associados. Cad Saúde Pública 2013; 29:335-48.

29. Godoy KC, Sávio KEO, Akutsu RC, Gubert MB, Botelho RBA. Perfil e situação de insegurança alimentar dos usuários dos Restaurantes Populares no Brasil. Cad Saúde Pública 2014; 30:1239-49.

30. Hoffmann R. Transferências de renda e desigualdades no Brasil (1995-2011). In: Campello T, Neri MC, organizadores. Programa Bolsa Família: uma década de inclusão e cidadania. Brasília: Instituto de Pesquisa Econômica Aplicada; 2013. p. 207-16.

31. Instituto Brasileiro de Análises Sociais e Econômicas. Repercussões do Programa Bolsa Família na segurança alimentar e nutricional das famílias beneficiadas. Rio de Janeiro: Instituto Brasileiro de Análises Sociais e Econômicas; 2008. 
32. Cabral CS, Lopes AG, Lopes JM, Vianna RPT. Segurança alimentar, renda e Programa Bolsa Família: estudo de coorte em municípios do interior da Paraíba, Brasil, 2005-2011. Cad Saúde Pública 2014; 30:393-402.

33. Fachine LA, Nunes BP, Motta JVS, Tomasi E, Silva SM, Thumé E, et al. Insegurança alimentar no Nordeste e Sul do Brasil: magnitude, fatores associados e padrões de renda per capita para redução das iniquidades. Cad Saúde Pública 2014; 30:161-74.

\section{Abstract}

This study aimed to measure the prevalence of food insecurity in a rural area of Northeast Brazil and investigate this outcome according to residence in quilombola communities (descendants of African slaves) versus non-quilombola communities. This was a cross-sectional study in 21 rural communities, 9 of which quilombolas, in 2014, using the Brazilian Food Insecurity Scale (EBIA). Prevalence rates and prevalence ratios were estimated for food insecurity, and Poisson multiple regression analysis with robust variance was performed. Food insecurity was found in $52.1 \%$ of the families: $64.9 \%$ in quilombola communities and $42 \%$ in the others. Food insecurity was associated with belonging to a quilombola community $(P R=$ 1.25), lower economic status $(P R=1.89 ; 2.98$, and 3.22 for status $C 2, D$, and $E$, respectively), beneficiaries of Bolsa Familia Program $(P R=1.52)$, and four or more household members $(P R=1.20)$. Food insecurity prevalence was high in the entire population, but it was even higher in quilombola communities, even though they belonged to the same coverage area. The results emphasize this population's vulnerability.

Food and Nutrition Security; African Continental Ancestry Group; Ethnicity and Health;

Rural Population
34. Cotta RMM, Machado JC. Programa Bolsa Família e segurança alimentar e nutricional no Brasil: revisão crítica da literatura. Rev Panam Salud Pública 2013; 33:54-60.

35. Lignani JB, Sichieri R, Burlandy L, Salles-Costa R. Changes in food consumption among the Programa Bolsa Família participant families in Brazil. Public Health Nutr 2010; 14:785-92

\section{Resumen}

Este artículo tuvo como objetivo identificar la prevalencia de la inseguridad alimentaria en un área rural del Nordeste de Brasil e investigar este resultado, de acuerdo con la residencia en comunidades quilombolas y no quilombolas. Fue un estudio transversal con 21 comunidades rurales, siendo 9 quilombolas, en 2014, utilizando Escala Brasileña de Inseguridad Alimentaria (EBIA). Se estimaron prevalencias y razones de prevalencia para inseguridad alimentaria, y el análisis múltiple fue dirigido por regresión de Poisson con variancia robusta. La situación de inseguridad alimentaria se encontró en un 52,1\% de las familias estudiadas, siendo un 64,9\% entre quilombolas y un $42 \%$ entre las demás. Inseguridad alimentaria se asoció a ser quilombola $(R P=1,25)$; tener un nivel económico más bajo $(R P=1,89 ; 2,98$ y 3,22 para los niveles C2, D y E, respectivamente); ser beneficiario del programa Beca Familia $(R P=1,52)$; y la existencia de 4 residentes o más en el domicilio $(R P=$ 1,20). La prevalencia de inseguridad alimentaria se elevó en toda la población, no obstante, las comunidades quilombolas, a pesar de pertenecer a la misma área de alcance de las otras comunidades, presentaron una prevalencia incluso mayor de inseguridad alimentaria, reforzando la vulnerabilidad de esa población.

Seguridad Alimentaria y Nutricional; Grupo de Ascendencia Continental Africana; Origen Étnico y Salud; Población Rural
Recebido em 13/Jan/2016

Versão final reapresentada em 25/Mai/2016

Aprovado em 24/Jun/2016 